\title{
Enough is enough
}

\section{Christine Borowski}

Executive Editor, The Journal of Experimental Medicine

Complaints about the overabundance of supplementary information in primary research articles have increased in decibel and frequency in the past several years and are now at cacophonous levels. Reviewers and editors warn that they do not have time to scrutinize it. Authors contend that the effort and money needed to produce it exceeds that reasonably spent on a single publication. How often readers actually look at supplemental information is unclear, and most journal websites offer the supplement as an optional download.

The abundance of supplemental material in The Journal of Experimental Medicine is growing. In the May 2011 issue of JEM, all research papers have a supplement, with an average of 5.9 supplementary items per paper. Only 3 yr ago (May 2008 issue), 16\% of JEM papers had no supplementary items, and the overall average was 4.6 items per paper. $3 \mathrm{yr}$ before that (May 2005 issue), 57\% of papers had no supplementary items, and the average was only 2.4 items per paper.

Why the increase in the prevalence of supplementary data? Reviewers frequently ask for it. Editors generally allow it. So authors are compelled to provide it, although some do so grudgingly. Of course, authors are also referees. Why the same individual would demand experiments as a referee that they might balk at as an author seems paradoxical.

What we can do to save authors and referees from this supplemental surplus is create a policy to address the problem. Effective immediately, supplementary information in JEM Articles and Brief Definitive Reports (BDRs) will be limited to essential supporting information. This would include: file formats not currently amenable to inclusion in the primary paper (e.g., videos, large datasets, and genomic data), clinical information (e.g., case reports and descriptions of patient populations), and complex detailed methods required to reproduce data in the paper (e.g., chemistry required to synthesize and characterize compounds, evidence of the purity and specificity of new inhibitors or antibodies, or flow cytometry gating strategies).

Our aim is to eliminate the use of the supplement as a "data dump." For example, additional replicates of experiments shown in the primary paper will no longer be included in the supplement. Data documenting that a commercially available antibody does in fact deplete cells bearing its target, as shown by previous publications, will no longer be in the supplement. In some cases, especially with BDRs, the editors may decide that data unraveling the mechanism underpinning a reported phenomenon or phenotype are beyond the scope of the current manuscript. In these cases, authors will not be required to include data providing a preliminary "hint" at a mechanism in the supplement.

Although we encourage authors to format new manuscripts in accordance with this new policy, JEM will not enforce supplementary information limits at the time of submission. We will, however, provide detailed guidance to authors of manuscripts that are invited back after external review.

Referees will be reminded of this new policy in the referee instructions letter accompanying each manuscript. However, we will inevitably encounter situations in which referees request substantial amounts of new experimental data. In these scenarios, the editors will provide authors with explicit guidance. In some cases, we may feel that the requested data are beyond the scope of the current manuscript. In others, the editors may agree that a large amount of new data would be needed to raise the conceptual scope and novelty of the manuscript. In the latter situation, authors would need to refocus the paper rather than simply submit a revised paper that is twice its original

Effective immediately, supplementary information in JEM Articles and BDRs will be limited to essential supporting information.

size. In some cases, papers originally submitted as BDRs can be reformatted as full Articles. However, we realize that in many instances the extent of required refocusing may ultimately result in the generation of a manuscript that tells a distinctly different story from the original. We will emphasize this point in our communication with authors.

The intention of the new policy is to curtail the escalating demand on the time and effort of authors, referees and editors, and to expedite the publication of exciting new findings. As with all policy changes, we welcome your feedback and suggestions.

(- 2011 This article is distributed under the terms of an Attribution Noncommercial-Share Alike-No Mirror Sites license for the first six months after the publication date (see http://www.rupress.org/ terms). After six months it is available under a Creative Commons License (Attribution-Noncommercial-Share Alike 3.0 Unported license, as described at http://creativecommons.org/licenses/by-nc-sa/3.0/). 